\title{
Improved Path Planning and Attitude Control Method for Agile Maneuver Satellite with Double-Gimbal Control Moment Gyros
}

\author{
Peiling Cui, ${ }^{1,2}$ Jingxian He, ${ }^{1,2}$ Jian Cui, ${ }^{1,2}$ and Haitao $\mathrm{Li}^{1,2}$ \\ ${ }^{1}$ School of Instrumentation Science and Optoelectronics Engineering, Beihang University, Beijing 100191, China \\ ${ }^{2}$ Science and Technology on Inertial Laboratory, Beijing 100191, China \\ Correspondence should be addressed to Peiling Cui; cuiplhh@126.com
}

Received 4 November 2014; Revised 2 February 2015; Accepted 8 February 2015

Academic Editor: Mohamed Abd El Aziz

Copyright (c) 2015 Peiling Cui et al. This is an open access article distributed under the Creative Commons Attribution License, which permits unrestricted use, distribution, and reproduction in any medium, provided the original work is properly cited.

\begin{abstract}
Double-gimbal control moment gyros can implement the satellite attitude maneuver efficiently. In order to reduce the energy consumption of double-gimbal control moment gyros and avoid the singularity state, an attitude maneuver path planning method is proposed by using the improved Fourier basis algorithm. Considering that the choice of the Fourier coefficients is important for the Fourier basis algorithm to converge quickly, a choosing method of the initial Fourier coefficients which can reduce the computational time of the path planning algorithm notably is proposed. Moreover, an attitude-tracking feedback controller based on the Fourier basis path planning algorithm is designed to acquire robustness. Simulation results show that the proposed path planning algorithm can implement attitude maneuver path planning in shortened time. Meanwhile, the feasibility of the attitude-tracking feedback controller which is based on the above path planning algorithm is verified in terms of the low energy consumption, high attitude-tracking precision, and the safe use of double-gimbal control moment gyros.
\end{abstract}

\section{Introduction}

High-resolution earth observation demands the satellite to have the ability of rapid attitude maneuver. Therefore, control moment gyros (CMGs) which can output large and precise torque are ideal attitude control actuator for the agile maneuver satellite. The main problem of using CMGs is the existence of singularity state, at which CMGs cannot produce torque in a certain direction. According to the gimbal numbers, CMGs can be divided into two classes, including single-gimbal CMGs (SGCMGs) and double-gimbal CMGs (DGCMGs). The singularity avoidance steering law is very important for SGCMGs to fulfill the requirement of the rapid attitude maneuver. Compared with SGCMG, DGCMG has more than one degree of freedom, and its angular momentum envelop is nearly spherical. Therefore, it is believed that DGCMGs can implement the rapid attitude maneuver with high efficiency.

Existing attitude control logics with CMGs are composed of attitude control laws and CMG steering laws $[1,2]$. According to attitude tracking errors, the desired attitude control torque is computed by the attitude control laws.
The objective of the CMG steering laws is to avoid singularity states. A large-angle attitude maneuver control law based on nonlinear methods is proposed in [1], and a singularity robustness plus null motion steering law is designed to ensure DGCMGs against singularity. References $[3,4]$ investigate on the singularity avoidance steering law specially. A modified singular-direction avoidance steering law of SGCMGs is introduced in [3]. Kennel presents the vector distribution steering law for parallel-mounted DGCMGs in [4], which makes use of redundant degrees of freedom of DGCMGs.

However, when the CMG steering law is separated from the attitude control law, CMG singularity problem and energy consumption cannot be considered in the computing process of attitude control torque. In addition, there exist singularity states which cannot be avoided by CMG steering laws. To deal with the above problems, the attitude control and CMG steering law should be integrated together as a whole control system.

An effective method of integrating the attitude control law and CMG steering law together is to plan the attitude maneuver path according to the satellite maneuver characteristic. 
Recent path planning algorithms mainly include Legendre pseudospectral algorithm $[5,6]$, generic algorithm (GA) $[7$, $8]$, and Fourier basis algorithm (FBA) [9, 10]. By using an integral cost function consisting of energy consumption and time cost index, [5] applies the Legendre pseudospectral algorithm to the optimal path planning. Reference [6] reviews key theoretical results of the pseudospectral optimal method which are important for successful flights. An energy optimal attitude maneuver path is planned by using the improved GA [7].

The Legendre pseudospectral algorithm and generic algorithm have many optimal parameters, thus resulting in complex computation. Moreover, the two algorithms generate time-discrete control inputs, which need to be approximated by continuous functions after the path planning. The rapid attitude maneuver control systems of agile satellites are real-time systems. Therefore, the Legendre pseudospectral algorithm and generic algorithm are not very suitable for the rapid attitude maneuver control. Regarding the gimbal rates of SGCMGs as control inputs, $[9,10]$ proposes a continuous optimal path planning method based on the FBA. The idea of FBA is to approximate control inputs by applying a Fourier series. The path planning method based on FBA is advantageous in terms of the computational time, and the path planning results are continuous. However, the Fourier coefficient vector is initialized by some random process in $[9,10]$, and the algorithm cannot converge rapidly. The choosing method of the initial Fourier coefficient has not been introduced yet. Moreover, there exists no Fourier basis path planning method which is proposed to investigate the attitude control of agile maneuver satellite with DGCMGs. Besides, since the disturbances and attitude tracking errors have not been considered in the path planning, the attitude tracking controller which can achieve the system robustness needs to be improved $[5,9]$.

This paper fills the above gap in the existing literature. The path planning and attitude control law of the agile maneuver satellite with DGCMGs are integrated together. The energy consumption and terminal state errors of doublegimbal control moment gyroscopes are considered at the same time. An improved FBA is proposed for the attitude maneuver path planning for agile maneuver satellite with DGCMGs. In order to shorten the computational time of the path planning, a choosing method of the initial Fourier coefficient is introduced. Meanwhile, the attitude tracking feedback controller based on the path planning method is proposed by applying a sliding mode controller. Simulations are carried out to verify the availability of the introduced path planning method and the corresponding attitude tracking controller.

\section{Model of the Satellite Attitude Control System}

As shown in Figure 1, the two parallel-mounted DGCMGs are used as the attitude control actuators. $\mathbf{x}_{b}, \mathbf{y}_{b}$, and $\mathbf{z}_{b}$ are unit vectors aligned along the axes of the satellite body frame coordinate. The orthonormal set of unit vectors $\mathbf{x}_{i}, \mathbf{y}_{i}, \mathbf{z}_{i}$ is used to describe the orientation of the $i$ th DGCMG relative to the spacecraft body frame, where $\mathbf{x}_{i}$ is fixed along the inner gimbal axis, $\mathbf{y}_{i}$ is fixed along the outer gimbal axis, and $\mathbf{z}_{i}$ is fixed along the wheel spin axis. $\alpha_{i}$ and $\beta_{i}$ denote the inner gimbal angle and outer gimbal angle of the $i$ th DGCMG, respectively. $\dot{\alpha}_{i}$ and $\dot{\beta}_{i}$ represent the inner gimbal rate and outer gimbal rate of the $i$ th DGCMG, respectively. Assuming that $h_{0}$ denote the wheel angular momentum of the DGCMG, the total angular momentum of two parallelmounted DGCMGs expressed in the satellite body frame coordinate can be written as

$$
\mathbf{h}_{\mathrm{cmg}}=h_{0}\left[\begin{array}{c}
\cos \alpha_{1} \cos \beta_{1}+\cos \alpha_{2} \cos \beta_{2} \\
-\cos \alpha_{1} \sin \beta_{1}-\cos \alpha_{2} \sin \beta_{2} \\
-\sin \alpha_{1}-\sin \alpha_{2}
\end{array}\right] \text {. }
$$

The output torque of DGCMGs can be obtained by using the time derivative of $\mathbf{h}_{\mathrm{cmg}}$ as follows:

$$
\begin{aligned}
\mathbf{T}_{\mathrm{cmg}} & =-\dot{\mathbf{h}}_{\mathrm{cmg}} \\
& =-h_{0}\left[\begin{array}{cccc}
-\sin \alpha_{1} \cos \beta_{1} & -\cos \alpha_{1} \sin \beta_{1} & -\sin \alpha_{2} \cos \beta_{2} & -\cos \alpha_{2} \sin \beta_{2} \\
\sin \alpha_{1} \sin \beta_{1} & -\cos \alpha_{1} \cos \beta_{1} & \sin \alpha_{2} \sin \beta_{2} & -\cos \alpha_{2} \cos \beta_{2} \\
-\cos \alpha_{1} & 0 & -\cos \alpha_{2} & 0
\end{array}\right] \dot{\boldsymbol{\delta}}=-h_{0} \mathbf{C}(\boldsymbol{\delta}) \dot{\boldsymbol{\delta}},
\end{aligned}
$$

where $\boldsymbol{\delta}=\left[\begin{array}{llll}\alpha_{1} & \beta_{1} & \alpha_{2} & \beta_{2}\end{array}\right]^{T}$ denotes the gimbal angle vector of two parallel-mounted DGCMGs and $\dot{\delta}$ is the time derivative of $\boldsymbol{\delta}$ with respect to the satellite body frame. $\mathrm{C}$ is a $3 \times 4$ matrix, which can describe the three-dimensional control authority of DGCMGs. As the singularity index $\operatorname{det}\left(\mathbf{C C}^{\mathbf{T}}\right)$ approaches zero, DGCMGs will encounter singularity.
Considering the angular momentum of each component in the satellite attitude control system, the total angular momentum is

$$
\mathbf{H}_{\mathbf{b}}=\mathbf{I}_{\mathbf{b}} \boldsymbol{\omega}+\mathbf{h}_{\mathrm{cmg}},
$$




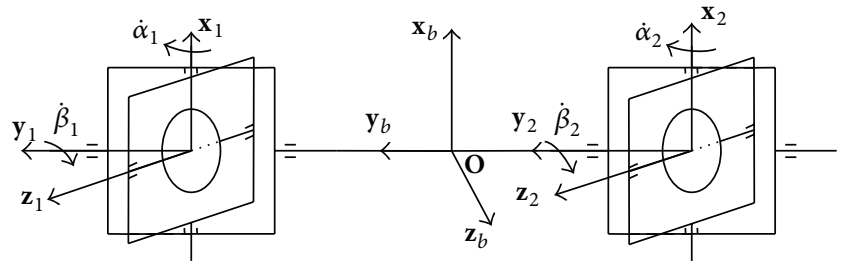

FIGURE 1: Illustration of the two parallel-mounted DGCMGs.

where $\mathbf{I}_{\mathbf{b}}$ represents the satellite moment of inertia and $\boldsymbol{\omega}$ represents the attitude angular velocity. Supposing that the satellite body is rigid, the dynamic equation is

$$
\dot{\mathbf{H}}_{b}+\boldsymbol{\omega} \times \mathbf{H}_{b}=\mathbf{T}_{d},
$$

where $\dot{\mathbf{H}}_{b}$ is the time derivative of $\mathbf{H}_{b}$ with respect to the satellite body frame and $\mathbf{T}_{d}$ is the disturbance toque. According to (2) and (3), (4) can be rewritten as

$$
\mathbf{I}_{\mathbf{b}} \dot{\boldsymbol{\omega}}+\boldsymbol{\omega} \times \mathbf{I}_{\mathbf{b}} \boldsymbol{\omega}+h_{0} \mathbf{C}(\boldsymbol{\delta}) \dot{\boldsymbol{\delta}}+\boldsymbol{\omega} \times \mathbf{h}_{\mathrm{cmg}}=\mathbf{T}_{d} .
$$

Aiming at describing the large-angle attitude maneuver, the quaternion kinematics equation yields

$$
\dot{\mathbf{q}}=\frac{1}{2}\left[\begin{array}{cccc}
0 & -\omega_{x} & -\omega_{y} & -\omega_{z} \\
\omega_{x} & 0 & \omega_{z} & -\omega_{y} \\
\omega_{y} & -\omega_{z} & 0 & \omega_{x} \\
\omega_{z} & \omega_{y} & -\omega_{x} & 0
\end{array}\right] \mathbf{q}=\mathbf{E}(\boldsymbol{w}) \mathbf{q}
$$

where $\mathbf{q}=\left[\begin{array}{ll}q_{0} & \overline{\mathbf{q}}\end{array}\right]^{T}$ is unit quaternion. $q_{0}$ and $\overline{\mathbf{q}}$ are scalar and vector components of q. $\omega_{x}, \omega_{y}$, and $\omega_{z}$ denote elements of $\boldsymbol{\omega}$ expressed in the satellite body frame coordinate. $\mathrm{E}$ is a $4 \times 4$ matrix associated with the attitude angular velocity.

Selecting $\mathbf{x}=\left[\begin{array}{ll}\mathbf{q}^{T} & \boldsymbol{\omega}^{T}\end{array}\right]^{T}$ as the state variable, the attitude control model of the satellite equipped with DGCMGs can be described as

$$
\begin{gathered}
\dot{\mathbf{x}}=\mathbf{f}(\boldsymbol{\delta}) \mathbf{u}+\mathbf{g}(\mathbf{x}, \boldsymbol{\delta}), \\
\mathbf{g}(\mathbf{x}, \boldsymbol{\delta})=\left[\begin{array}{c}
\mathbf{E}(\boldsymbol{\omega}) \mathbf{q} \\
-\mathbf{J}^{-1}\left(\boldsymbol{\omega} \times \mathbf{J} \boldsymbol{\omega}+\boldsymbol{\omega} \times \mathbf{h}_{\mathrm{cmg}}\right)
\end{array}\right], \\
\mathbf{f}(\boldsymbol{\delta})=\left[\begin{array}{c}
0 \\
-\mathbf{J}^{-1} \mathbf{C}(\boldsymbol{\delta}) h_{0}
\end{array}\right],
\end{gathered}
$$

where the control input vector $\mathbf{u}$ is the DGCMG gimbal rate vector $\dot{\delta}$. The boundary conditions of (7) are

$$
\mathbf{x}(0)=\mathbf{x}_{0}, \quad \mathbf{x}\left(t_{f}\right)=\mathbf{x}_{d}
$$

where $t_{f}$ is the desired attitude maneuver time. $\mathbf{x}_{0}$ is the initial state variable vector. $\mathbf{x}_{d}$ is the desired state variable vector.

\section{Path Planning Method Based on the Improved Fourier Basis Algorithm}

3.1. Introduction of the Improved Fourier Basis Algorithm. The path planning method based on the improved FBA adopts a Fourier series to approximate the control inputs. The searching methods, such as gradient descent method and Newton method, are applied to search for the near optimal Fourier coefficients, which make the cost function of the attitude path planning converge to its minimal value. In order to achieve the safe use of DGCMGs, the path planning objective is to obtain the smooth attitude maneuver path which keeps the maximal gimbal rate low, avoiding vibrating motions. Therefore, the energy consumption of DGCMGs should be low enough to meet the requirement of the path planning objective. By considering the terminal state errors and the energy consumption of DGCMGs, the cost function of the attitude maneuver path planning yields

$$
J(\mathbf{u})=\int_{0}^{t_{f}}\left(\mathbf{u}^{T} \mathbf{u}\right) d t+\left(\mathbf{x}\left(t_{f}\right)-\mathbf{x}_{d}\right)^{T} \mathbf{G}\left(\mathbf{x}\left(t_{f}\right)-\mathbf{x}_{d}\right),
$$

where $\mathbf{G}$ is a weighting matrix, which should be large enough to reduce the terminal state errors. The first term in the cost function is the energy cost of control inputs, and the second term depends on terminal state errors. If the control inputs are expressed by an orthonormal basis for $\mathbf{L}_{2}\left(\left[0, t_{f}\right]\right)$, the search for control inputs can be converted into solving for optimal parameters of the expression. By choosing a Fourier basis to describe the control input vector and restricting control inputs to the first three terms of the Fourier basis, the following can be obtained:

$$
\mathbf{u}=\phi \boldsymbol{\alpha},
$$

where

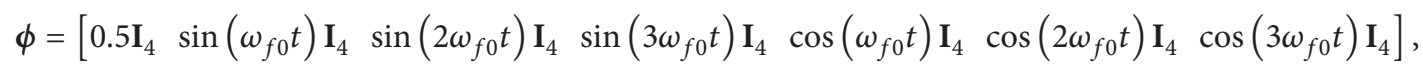

where $\phi$ denotes the Fourier base term matrix, $\boldsymbol{\alpha}$ denotes the Fourier coefficient vector, $\mathbf{I}_{4}$ is a $4 \times 4$ identity matrix, and $\omega_{f 0}=2 \pi / t_{f}$ is the basic frequency of the Fourier basis approximation. Inserting (12) into (11), the cost function of the path planning comes to depend on the Fourier coefficients as follows:

$$
J(\boldsymbol{\alpha})=\boldsymbol{\alpha}^{T} \boldsymbol{\alpha}+\left(\mathbf{x}\left(t_{f}\right)-\mathbf{x}_{d}\right)^{T} \mathbf{G}\left(\mathbf{x}\left(t_{f}\right)-\mathbf{x}_{d}\right) .
$$


In this way, the problem of the path planning based on FBA is equivalent to a nonlinear search problem for the near optimal coefficients that makes the cost function approach its minimal value.

3.2. Choosing Method of the Initial Fourier Coefficients. The choice of the initial Fourier coefficients is very important to make the path planning method based on improved FBA converge rapidly, which can improve the convergence speed of the algorithm. The initial Fourier coefficients are computed by the initial path of DGCMG gimbal rates. Moreover, as can be seen in (7), gimbal rates can be generated by the quaternion. Therefore, the choice of the initial Fourier coefficients can start with the determination of initial quaternion paths. $\left(\overline{\mathbf{q}}_{1}, \overline{\mathbf{q}}_{2}, \ldots, \overline{\mathbf{q}}_{n}\right)$ is used to denote the initial quaternion path, where $\overline{\mathbf{q}}_{1}$ and $\overline{\mathbf{q}}_{n}$ are the vector components of the initial quaternion and the target quaternion, respectively. $\overline{\mathbf{q}}_{j}(j=2, \ldots n-1)$ represents the vector components of the intermediate nodes of the quaternion path. The path length $n=t_{f} / T_{s}$ is calculated by the simulation time $t_{f}$ and simulation step $T_{s}$. According to (6), $\overline{\mathbf{q}}_{2}$ can be computed by the initial quaternion $\overline{\mathbf{q}}_{1}$ and initial attitude angular velocity $\boldsymbol{\omega}_{0}$. To meet the requirement of satellite attitude agile maneuver, intermediate nodes $\left(\overline{\mathbf{q}}_{3}, \ldots, \overline{\mathbf{q}}_{n-1}\right)$ in the initial quaternion path are produced based on the sigmoid function:

$$
\begin{array}{r}
\overline{\mathbf{q}}_{j}=\frac{\overline{\mathbf{q}}_{n}-\overline{\mathbf{q}}_{2}}{1+\exp ^{n}\left[-\left(c j T_{s}+(a-2) T_{s}-m\right)\right]}+\overline{\mathbf{q}}_{2} \\
j \in(3,4, \ldots, n-1),
\end{array}
$$

where $m$ is an appropriate constant. $a$ and $c$ can be computed as follows:

$$
\begin{gathered}
\mathbf{f}_{2}(t)=\frac{\overline{\mathbf{q}}_{n}-\overline{\mathbf{q}}_{2}}{1+\exp ^{n}[-(t-m)]}+\overline{\mathbf{q}}_{2}, \\
\Delta \mathbf{f}_{2}(j)=\mathbf{f}_{2}\left((j+1) T_{s}\right)-\mathbf{f}_{2}\left(j T_{s}\right), \\
a=\min \left(\operatorname{find}\left(\Delta \mathbf{f}_{2}>10^{-3}\right)\right), \\
c=\frac{b-a+1}{n-2}, \\
b=\max \left(\text { find }\left(\Delta \mathbf{f}_{2}>10^{-3}\right)\right),
\end{gathered}
$$

where $t \in\left(0, T_{a}\right)$ and $T_{a}$ is a constant larger than $t_{f}$.

When the quaternion path $\left(\overline{\mathbf{q}}_{1}, \overline{\mathbf{q}}_{2}, \ldots, \overline{\mathbf{q}}_{n}\right)$ is obtained, the attitude angular velocity can be achieved by the time derivative of quaternion. Furthermore, DGCMG gimbal rates can be calculated by the attitude angular velocity according to (5). Finally, the Fourier series shown in (13) is applied to approximating the evaluating gimbal rates, thus generating the initial Fourier coefficient vector.
3.3. Search Method for the Fourier Basis Algorithm. The Newton method based on the first and second partial derivatives of the cost function can improve the convergence property of the path planning algorithm. However, it is difficult to compute the second partial derivative of the cost function, and the above choosing method of the initial Fourier coefficient vector has improved the convergence speed already. Therefore, the gradient descent method which only based on the first partial derivative of the cost function is applied to searching for the near optimal Fourier coefficient vector. The computation of the first-order derivative of the cost function at the searching point $\boldsymbol{\alpha}_{n}$ is shown as follows:

$$
\frac{\partial \mathbf{J}\left(\boldsymbol{\alpha}_{n}\right)}{\partial \boldsymbol{\alpha}_{n}}=-\mu\left(\boldsymbol{\alpha}_{n}-\mathbf{y}^{T}\left(t_{f}\right) \mathbf{G}\left(\mathbf{x}-\mathbf{x}_{d}\right)\right),
$$

where $\mu$ is a positive constant and $\mathbf{y}\left(t_{f}\right)$ is described by

$$
\mathbf{y}\left(t_{f}\right)=\frac{\partial \mathbf{x}\left(t_{f}\right)}{\partial \boldsymbol{\alpha}}
$$

$\mathbf{y}\left(t_{f}\right)$ has no simple mathematic expression, and it can be numerically evaluated by integrating its time derivative $\dot{\mathbf{y}}(t)$ from $t=0$ to $t=t_{f} \cdot \dot{\mathbf{y}}(t)$ and $\mathbf{y}\left(t_{0}\right)$ are given as

$$
\dot{\mathbf{y}}(t)=\left(\sum_{i=1}^{4} \frac{\partial \mathbf{f}_{i}}{\partial \boldsymbol{\delta}} \mathbf{u}_{i}\right) \mathbf{z}+\mathbf{f} \phi+\frac{\partial \mathbf{g}}{\partial \mathbf{x}} \mathbf{y}+\frac{\partial \mathbf{g}}{\partial \boldsymbol{\delta}} \mathbf{z}, \quad \mathbf{y}\left(t_{0}\right)=\mathbf{0},
$$

where $\mathbf{z}$ is the partial derivative of gimbal rates with respect to the Fourier coefficient vector. Its time derivative and initial value are

$$
\dot{\mathbf{z}}=\boldsymbol{\phi}, \quad \mathbf{z}\left(t_{0}\right)=\mathbf{0} .
$$

By applying the gradient descent method, the Fourier coefficient variation $\Delta \boldsymbol{\alpha}$ about the searching point $\boldsymbol{\alpha}_{n}$ is given by

$$
\Delta \boldsymbol{\alpha}=-\mu\left(\boldsymbol{\alpha}_{n}-\mathbf{y}^{T}\left(t_{f}\right) \mathbf{G}\left(\mathbf{x}-\mathbf{x}_{d}\right)\right) .
$$

Thus, the updating equation of the Fourier coefficient vector can be obtained:

$$
\boldsymbol{\alpha}_{n+1}=\boldsymbol{\alpha}_{n}+\Delta \boldsymbol{\alpha}
$$

The updating operation of the Fourier coefficient vector should be continued, until the near optimal solution of the path planning satisfies the requirement of DGCMG energy consumption and terminal state accuracy at the same time. Results of the proposed path planning method are composed of the varying path of the DGCMG gimbal rates, quaternion, and attitude angular velocity.

\section{Attitude Tracking Feedback Controller Based on the Improved Path Planning}

4.1. Model of Attitude Tracking Error. The error quaternion $\mathbf{q}_{e}=\left[\begin{array}{ll}q_{e 0} & \overline{\mathbf{q}}_{e}\end{array}\right]^{T}$ can be computed by the desired quaternion 


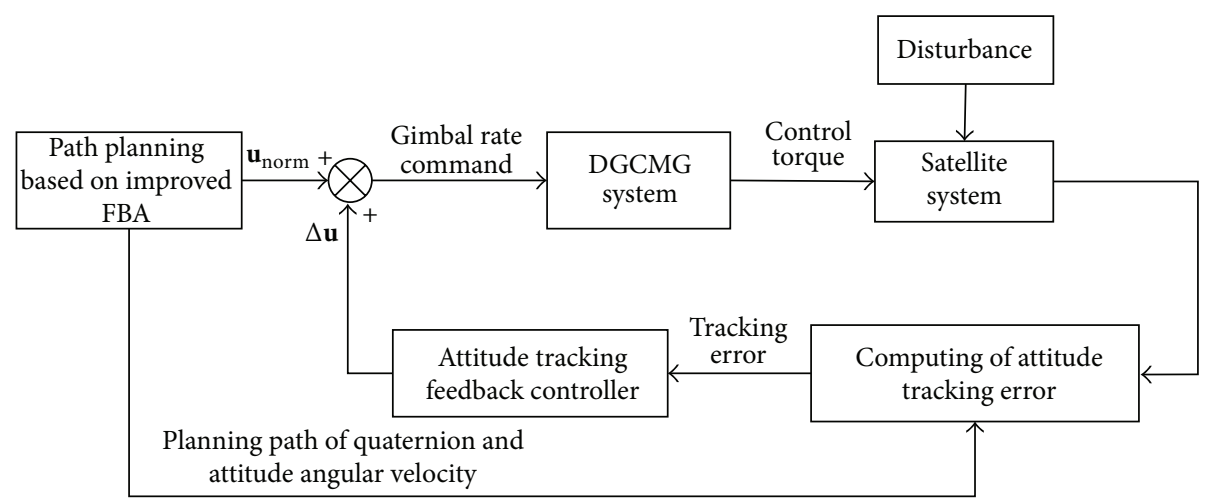

FIGURE 2: Block diagram of the attitude tracking feedback controller.

$\mathbf{q}_{d}=\left[\begin{array}{ll}q_{d 0} & \overline{\mathbf{q}}_{d}\end{array}\right]^{T}$ and the current quaternion $\mathbf{q}=\left[\begin{array}{ll}q_{0} & \overline{\mathbf{q}}\end{array}\right]^{T}$ as follows:

$$
\begin{gathered}
q_{e 0}=q_{0} q_{d 0}+\overline{\mathbf{q}}^{T} \overline{\mathbf{q}}_{d}, \\
\overline{\mathbf{q}}_{e}=q_{d 0} \overline{\mathbf{q}}-q_{0} \overline{\mathbf{q}}_{d}+\overline{\mathbf{q}} \times \overline{\mathbf{q}}_{d},
\end{gathered}
$$

where $q_{e 0}$ and $\overline{\mathbf{q}}_{e}$ have the constraint equation: $q_{e 0}^{2}+\overline{\mathbf{q}}_{e}^{T} \overline{\mathbf{q}}_{e}=1$. Assuming that $\omega_{d}$ is the desired attitude angular velocity, then the error of the attitude angular velocity is defined as

$$
\boldsymbol{\omega}_{e}=\boldsymbol{\omega}-\mathbf{C}_{d}^{b} \boldsymbol{\omega}_{d}
$$

where $\mathbf{C}_{d}^{b}=\left(q_{e 0}^{2}-\mathbf{q}_{e}{ }^{T} \mathbf{q}_{e}\right) \mathbf{I}_{3}+2 \mathbf{q}_{e} \mathbf{q}_{e}{ }^{T}-2 q_{e 0} \mathbf{q}_{e}{ }^{\times}, \mathbf{q}_{e}{ }^{\times}$is the cross matrix of $\mathbf{q}_{e}$, and $\mathbf{I}_{3}$ denotes $3 \times 3$ identity matrix.

By inserting (24) into (5), the following can be obtained:

$$
\mathbf{I}_{\mathbf{b}} \dot{\boldsymbol{\omega}}_{e}=\boldsymbol{\Gamma}\left(\mathbf{I}_{\mathbf{b}}, \boldsymbol{\omega}_{e}\right)-h_{0} \mathbf{C}(\boldsymbol{\delta}) \mathbf{u}+\mathbf{T}_{d},
$$

where $\boldsymbol{\Gamma}\left(\mathbf{I}_{\mathbf{b}}, \boldsymbol{\omega}_{e}\right)=-\left(\boldsymbol{\omega}_{e}+\mathbf{C}_{d}^{b} \boldsymbol{\omega}_{d}\right) \times\left[\mathbf{I}_{\mathbf{b}}\left(\boldsymbol{\omega}_{e}+\mathbf{C}_{d}^{b} \boldsymbol{\omega}_{d}\right)+\mathbf{h}_{\mathrm{cmg}}\right]+$ $\mathbf{I}_{\mathbf{b}}\left(\boldsymbol{\omega}_{e} \times \mathbf{C}_{d}^{b} \boldsymbol{\omega}_{d}-\mathbf{C}_{d}^{b} \dot{\boldsymbol{\omega}}_{d}\right)$.

According to the quaternion kinematics (6), the kinematics equation based on error quaternion can be written as

$$
\begin{gathered}
\dot{q}_{e 0}=-\frac{1}{2} \overline{\mathbf{q}}_{e}^{T} \boldsymbol{\omega}_{e}, \\
\dot{\overline{\mathbf{q}}}_{e}=\frac{1}{2} \mathbf{Z}\left(\overline{\mathbf{q}}_{e}\right) \boldsymbol{\omega}_{e},
\end{gathered}
$$

where $\mathbf{Z}\left(\overline{\mathbf{q}}_{e}\right)=q_{e 0} \mathbf{I}_{3}+\overline{\mathbf{q}}_{e} \times$.

4.2. Design of the Attitude Tracking Controller Based on the Improved Path Planning. The path planning method can acquire the continuous path of DGCMG gimbal rates which are effective in smooth action. The feedforward controller using the planning path of gimbal rates directly can track the desired quaternion path accurately without considering the disturbances. However, there always exist several disturbances, and the feedforward controller cannot maintain precise attitude control. In order to acquire the robustness against disturbances, the attitude tracking feedback controller is introduced based on the attitude maneuver path planned by the improved FBA. Since sliding mode controller has strong robustness and favorable dynamic response performance, the sliding mode controller is applied to the design of attitude tracking feedback controller. Moreover, the planning paths of the quaternion and attitude angular velocity are regarded as the tracking paths of the attitude maneuver. The objective of the feedback controller design is to compensate for the attitude tracking error by adding $\Delta \mathbf{u}$ to $\mathbf{u}_{\text {norm }}$, where $\mathbf{u}_{\text {norm }}$ represents the planning gimbal rate of the proposed path planning method and $\Delta \mathbf{u}$ is the added control input vector which can eliminate errors. The block diagram of the attitude tracking feedback controller is illustrated by Figure 2 .

The sliding surface $\mathbf{S}$ is chosen as

$$
\mathbf{S}=\omega_{e}+\mathbf{K} \overline{\mathbf{q}}_{e},
$$

where $\mathbf{K}$ is a positive diagonal matrix, $\omega_{e}$ is the error between the real angular velocity and the planning angular velocity, and $\overline{\mathbf{q}}_{e}$ is the vector component of error quaternion.

In order to verify the stability of the sliding mode $\mathbf{S}=\mathbf{0}$, the Lyapunov function $V_{1}$ is selected as follows:

$$
V_{1}=\frac{1}{2} \overline{\mathbf{q}}_{e}^{T} \overline{\mathbf{q}}_{e}+\frac{1}{2}\left(1-q_{e 0}\right)^{2}=1-q_{e 0} .
$$

It is obvious that $V_{1}$ is positive, and its time derivative is

$$
\dot{V}_{1}=-\dot{q}_{e 0}=\frac{1}{2} \overline{\mathbf{q}}_{e}^{T} \boldsymbol{\omega}_{e} .
$$

Inserting $\mathbf{S}=\mathbf{0}$ into (29), the following can be obtained:

$$
\dot{V}_{1}=-\frac{1}{2}\left(\mathbf{K}^{-1}\right)^{T} \boldsymbol{\omega}_{e}^{T} \boldsymbol{\omega}_{e} .
$$

Since $\mathbf{K}$ is a positive matrix, there exists $\dot{V}_{1}<0$, thus guaranteeing the stability of the sliding mode. The time derivative of $\mathbf{S}$ is

$$
\dot{\mathbf{S}}=\mathbf{I}_{\mathbf{b}}^{-1}\left(\boldsymbol{\Gamma}\left(\mathbf{I}_{\mathbf{b}}, \boldsymbol{\omega}_{e}\right)-h_{0} \mathbf{C}(\boldsymbol{\delta}) \mathbf{u}_{\mathbf{l}}+\mathbf{T}_{d}\right)+\frac{1}{2} \mathbf{K Z}\left(\overline{\mathbf{q}}_{e}\right) \boldsymbol{\omega}_{e},
$$

where $\mathbf{u}_{1}=\mathbf{u}_{\text {norm }}+\Delta \mathbf{u}$ is the total control input vector.

In order to generate a proper control law, another Lyapunov function $V_{2}$ is chosen as

$$
V_{2}=\frac{1}{2} \mathbf{S}^{T} \mathbf{I}_{\mathbf{b}} \mathbf{S} .
$$




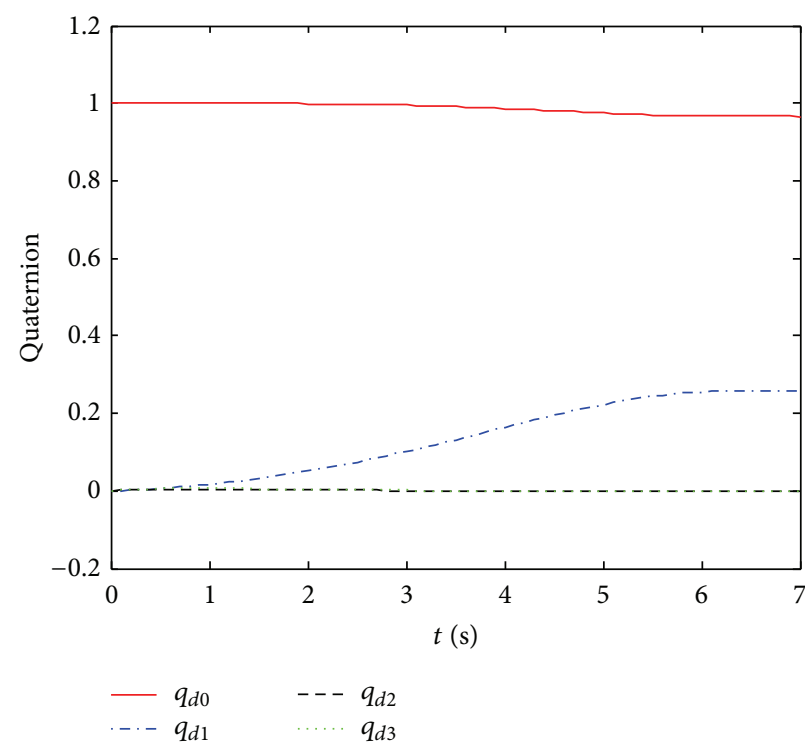

(a)

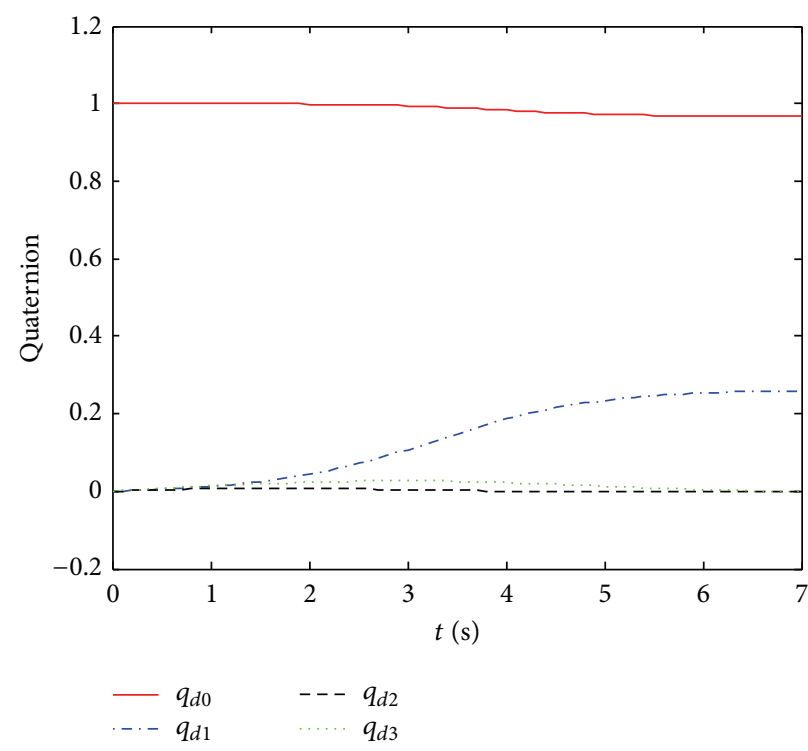

(b)

FIGURE 3: Time histories of quaternion. (a) Based on the path planning method in [7]. (b) Based on the path planning method in this paper.

The time derivative of $V_{2}$ can be written as

$$
\begin{aligned}
\dot{V}_{2} & =\mathbf{S}^{T} \mathbf{I}_{\mathbf{b}} \dot{\mathbf{S}} \\
& =\mathbf{S}^{T}\left(\boldsymbol{\Gamma}\left(\mathbf{I}_{\mathbf{b}}, \boldsymbol{\omega}_{e}\right)-h_{0} \mathbf{C}(\boldsymbol{\delta}) \mathbf{u}_{1}+\mathbf{T}_{d}+\frac{1}{2} \mathbf{I}_{\mathbf{b}} \mathbf{K Z}\left(\overline{\mathbf{q}}_{e}\right) \boldsymbol{\omega}_{e}\right) .
\end{aligned}
$$

Let

$$
\begin{aligned}
\mathbf{u}_{\mathbf{1}}= & \left(h_{0} \mathbf{C}(\boldsymbol{\delta})\right)^{-1} \\
& \cdot\left(\boldsymbol{\Gamma}\left(\mathbf{I}_{\mathbf{b}}, \boldsymbol{\omega}_{e}\right)+\mathbf{T}_{d}+\frac{1}{2} \mathbf{I}_{\mathbf{b}} \mathbf{K Z}\left(\overline{\mathbf{q}}_{e}\right) \boldsymbol{\omega}_{e}+\mathbf{K}_{g} \mathbf{S}\right. \\
& \left.+\mathbf{K}_{\beta} \operatorname{sign}(\mathbf{S})\right) .
\end{aligned}
$$

Then (33) can be rewritten as

$$
\dot{V}_{2}=-g \mathbf{S}^{T} \mathbf{S}-\beta \mathbf{S}^{T} \operatorname{sign}(\mathbf{S})
$$

where $\mathbf{K}_{g}$ and $\mathbf{K}_{\beta}$ are both positive diagonal matrices. It is obvious that $\dot{V}_{2}<0$, and the sliding surface can be reachable with the control law.

The saturation function is defined as

$$
\operatorname{sat}(\mathbf{S}, \xi)= \begin{cases}1 & \mathbf{S}>\xi \\ \frac{\mathbf{S}}{\xi} & |\mathbf{S}| \leq \xi \\ -1 & \mathbf{S}<-\xi\end{cases}
$$

where $\xi$ is a small positive constant. Aiming at avoiding the vibrating motions, the sign function in (34) is substituted by the saturation function as follows:

$$
\begin{aligned}
\mathbf{u}_{\mathbf{l}}= & \left(h_{0} \mathbf{C}(\boldsymbol{\delta})\right)^{-1} \\
& \cdot\left(\boldsymbol{\Gamma}\left(\mathbf{I}_{\mathbf{b}}, \boldsymbol{\omega}_{e}\right)+\mathbf{T}_{d}+\frac{1}{2} \mathbf{I}_{\mathbf{b}} \mathbf{K Z}\left(\overline{\mathbf{q}}_{e}\right) \boldsymbol{\omega}_{e}+\mathbf{K}_{g} \mathbf{S}+\mathbf{K}_{\beta} \operatorname{sat}(\mathbf{S})\right) .
\end{aligned}
$$

Then the added control input vector can be calculated by

$$
\Delta \mathbf{u}=\mathbf{u}_{1}-\mathbf{u}_{\text {norm }} .
$$

As can be seen from (37) and (38), the added control input vector $\Delta \mathbf{u}$ is associated with the attitude tracking errors, thus enabling it to compensate for the existing errors. It is confirmed that the added control inputs can improve the accuracy of the attitude control system.

\section{Simulations and Discussions}

Simulations for the path planning method based on improved FBA and the associated attitude tracking feedback controller are performed by using two parallel-mounted DGCMGs. The satellite moment of inertia is

$$
\mathbf{J}=\left[\begin{array}{ccc}
12 & 0.12 & 0.12 \\
0.13 & 13 & 0.13 \\
0.15 & 0.15 & 15
\end{array}\right] \mathrm{kg} \cdot \mathrm{m}^{2}
$$

Related parameters of the satellite and DGCMGs are shown as follows: the desired attitude maneuver time $t_{f}=$ $7 \mathrm{~s}$, the simulation step $T_{s}=0.1 \mathrm{~s}$, the initial quaternion $\mathbf{q}_{1}=\left[\begin{array}{llll}1 & 0 & 0 & 0\end{array}\right]^{T}$, the desired quaternion $\mathbf{q}_{d}=$ 


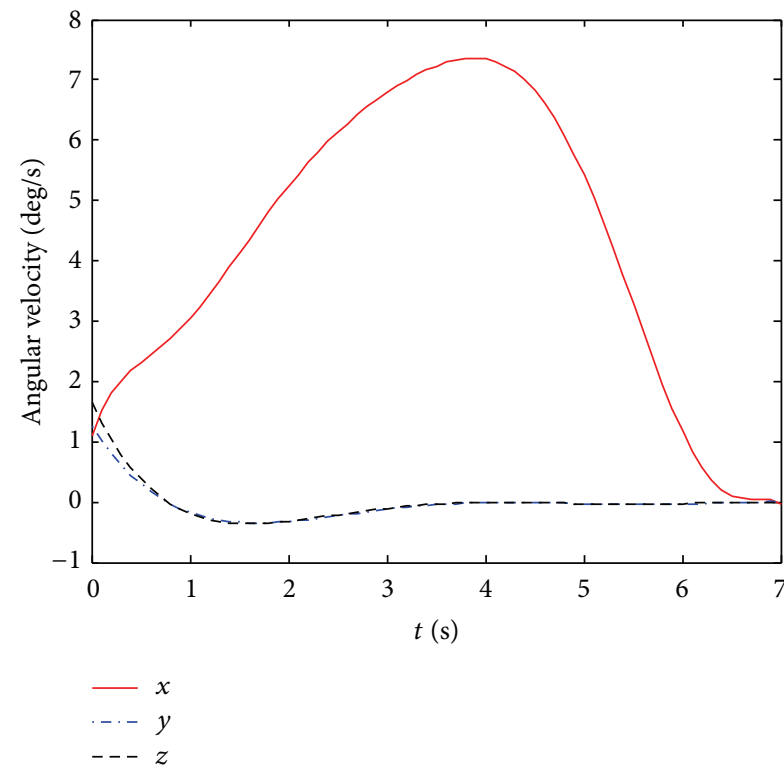

(a)

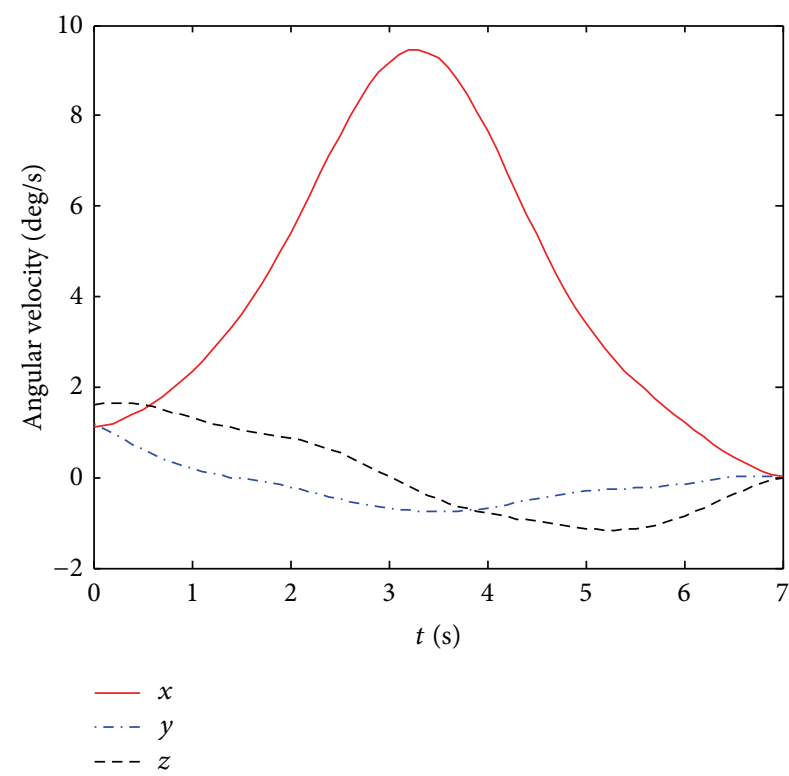

(b)

FIGURE 4: Time histories of attitude angular velocity. (a) Based on the path planning method in [7]. (b) Based on the path planning method in this paper.

$\left[\begin{array}{llll}0.97 & 0.26 & 0 & 0\end{array}\right]^{T}$, the initial attitude angular velocity $\boldsymbol{\omega}_{0}=$ $\left[\begin{array}{lll}1.1 & 1.2 & 1.6\end{array}\right]^{T} \mathrm{deg} / \mathrm{s}$, the desired attitude angular velocity $\boldsymbol{\omega}_{d}=\left[\begin{array}{lll}0 & 0 & 0\end{array}\right]^{T} \mathrm{deg} / \mathrm{s}$, the wheel angular momentum of DGCMGs $h_{0}=6 \mathrm{Nms}$, the initial gimbal angle vector $\boldsymbol{\delta}_{0}=$ $\left[\begin{array}{llll}0 & 0 & 0 & -90\end{array}\right]^{T} \mathrm{deg}$, and the maximal gimbal rate is $10 \mathrm{deg} / \mathrm{s}$.

5.1. Path Planning Results Based on the Improved FBA. Path planning results based on the improved FBA are compared with that in [7]. During the comparison, the related parameters of the satellite and DGCMGs are all the same. Parameters of the initial Fourier coefficient choosing method are $m=$ $8.05, n=1.96$. Parameters of searching method are $\mathbf{G}=$ $\operatorname{diag}(0,4,4,4,6,6,6) \times 10^{2}, \mu=2.2 \times 10^{-4}$. The stop condition of the Fourier coefficient update is $\left\|\mathbf{q}_{e}\right\|_{\infty} \leq 0.001,\left\|\boldsymbol{\omega}_{e}\right\|_{\infty} \leq$ $0.05 \mathrm{deg} / \mathrm{s}$. The DGCMG energy consumption in the path planning method is calculated by the following equation:

$$
\int_{0}^{t_{f}} \mathbf{u}^{T} \mathbf{u} d t=\int_{0}^{t_{f}} \dot{\boldsymbol{\delta}}^{T} \dot{\boldsymbol{\delta}} d t
$$

Figures 3-7 show the attitude maneuver path planning results based on the path planning method in [7] and that proposed in this paper. Figure 3 illustrates the varying path of the quaternion. Figure 4 illustrates the varying path of the attitude angular velocity. As can be seen from Figure 4, the attitude angular velocity of the both methods can converge to the desired value in the given maneuver time. Figure 4 illustrates the varying path of the attitude angular velocity.

Figure 5 gives the planning result of the DGCMG gimbal rates. As shown in Figure 5(a), DGCMG gimbal rates of the path planning method in [7] nearly reach the limit of $10 \mathrm{deg} / \mathrm{s}$. In contrast, the proposed improved path planning method can keep DGCMG gimbal rates under $8.2 \mathrm{deg} / \mathrm{s}$; thus, the safe use of DGCMGs is maintained, as shown in Figure 5(b). Furthermore, the initial gimbal rates of the path planning method in [7] reach $8.2 \mathrm{deg} / \mathrm{s}$. However, the initial gimbal rates of the proposed improved path planning are no more than $0.8 \mathrm{deg} / \mathrm{s}$. Since the tracking speed of the gimbal rate controller is limited, vibration cannot be avoided when the initial gimbal rates are large. The DGCMG gimbal angles are shown in Figure 6. All of the gimbal angles vary smoothly.

Figure 7 describes the singularity index of the two path planning methods. The minimal value of the singularity index in Figure 7(b) is larger than that in Figure 7(a). It indicates that the proposed method can plan effective paths which prevent DGCMGs from encountering singularity states. The computational time of the path planning method in [7] is about $30.4 \mathrm{~s}$. In contrast, the proposed method based on FBA can implement the path planning within $3.2 \mathrm{~s}$, and its computational time is $90 \%$ shorter than that of the method in [7]. Moreover, the DGCMG energy consumption of the proposed method is 0.0805 , which is $20 \%$ lower than that of the method in [7]. In a word, the proposed path planning method based on FBA is more effective than the method in [7] in the aspect of the computational time and DGCMG energy consumption.

5.2. Attitude Tracking Feedback Controller Based on the Improved FBA. Simulations for the attitude tracking controllers are carried out in this section. Results of the feedback controller have been compared with that of the feedforward controller which uses the path planning directly. Because the simulation time is much shorter than the varying period of the environmental disturbance, the disturbance torque vector is regarded as constant: $\mathbf{T}_{d}=\left[\begin{array}{lll}0.02 & 0.04 & 0.01\end{array}\right]^{T} \mathrm{Nm}$. 


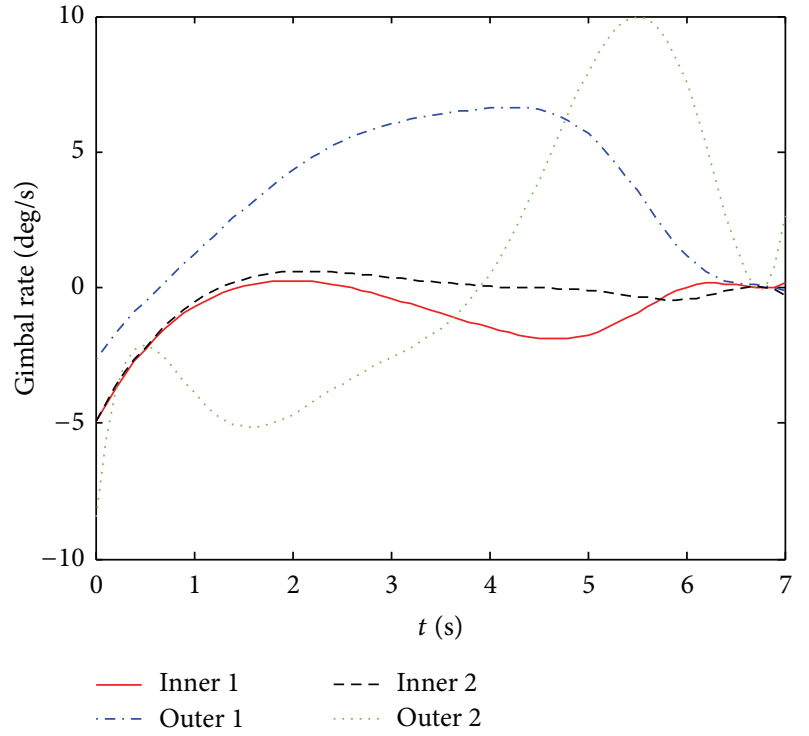

(a)

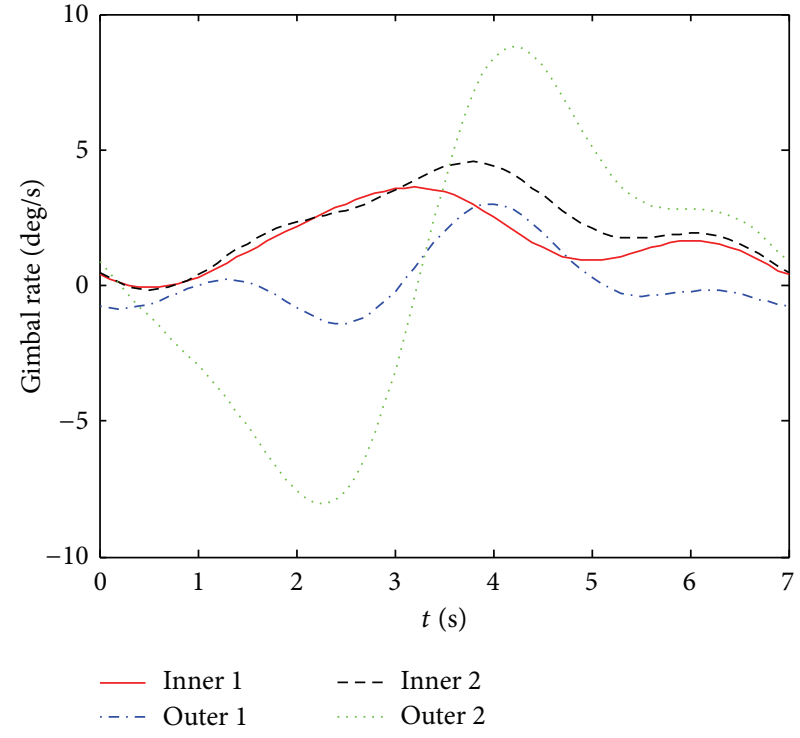

(b)

FIGURE 5: Time histories of gimbal angle velocity. (a) Based on the path planning method in [7]. (b) Based on the path planning method in this paper.

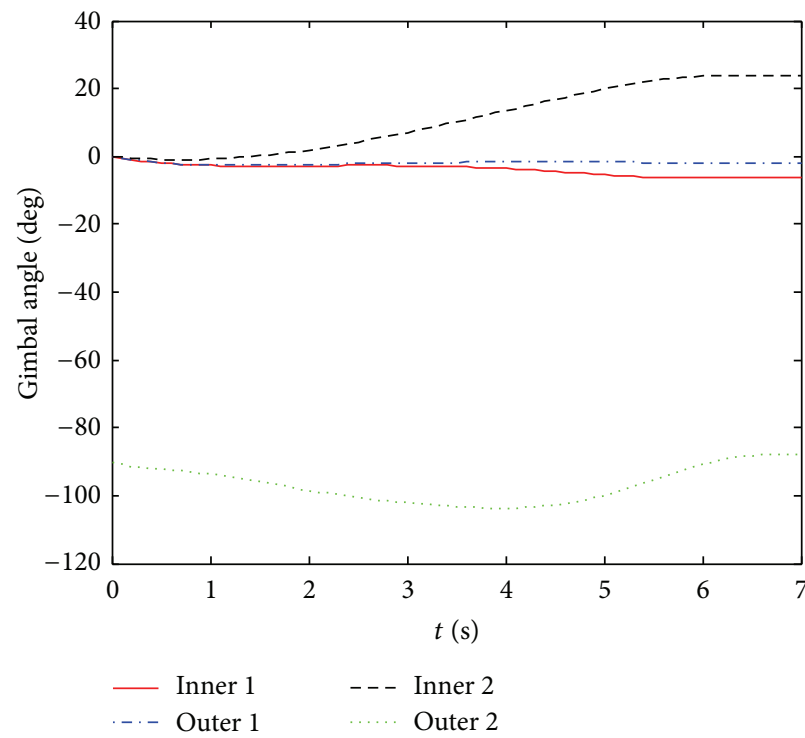

(a)

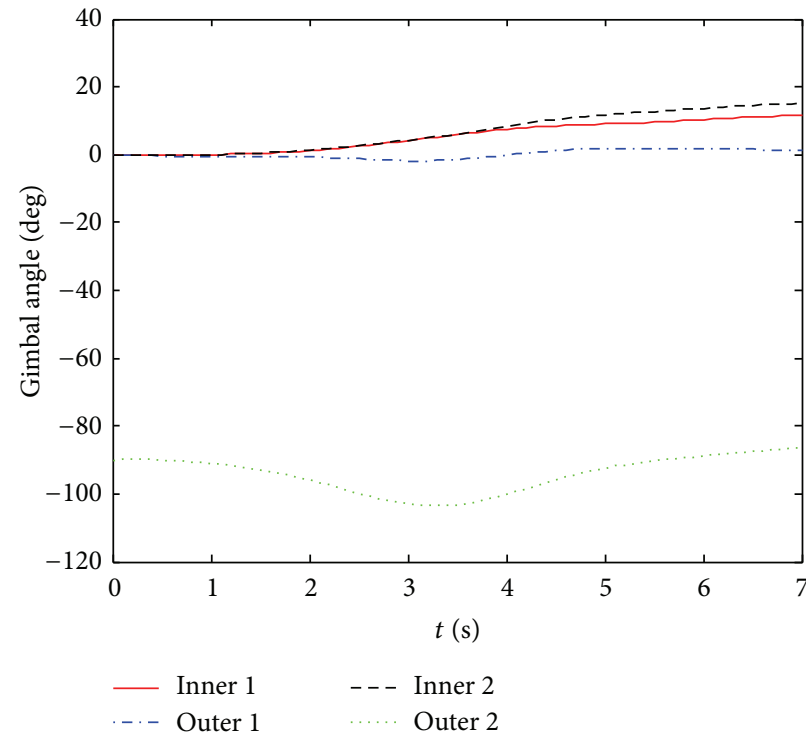

(b)

FIGURE 6: Time histories of gimbal angle. (a) Based on the path planning method in [7]. (b) Based on the path planning method in this paper.

Parameters of the attitude tracking feedback controller are chosen as $\mathbf{K}=\operatorname{diag}(2,1.2,1.2), \mathbf{K}_{\mathbf{g}}=\operatorname{diag}(5,8,8)$, and $\mathbf{K}_{\beta}=$ $\operatorname{diag}(1.0,1.2,1.2), \xi=0.1$.

Figures 8-11 give the simulation results of the feedforward and feedback controllers. Figure 8 illustrates the attitude quaternion trajectory. Figure 9 illustrates the attitude angular velocity trajectory. Figure 9(a) shows that the attitude angular velocity of the feedforward controller is still larger than $2 \times$ $10^{-2} \mathrm{rad} / \mathrm{s}$ at the terminal time, and the attitude maneuver has not been finished in the desired maneuver. On the other hand, the attitude angular velocity in Figure 9(b) is within $10^{-3} \mathrm{rad} / \mathrm{s}$ at the terminal time; thus, the feedback controller implements the attitude maneuver task.

Figure 10 illustrates the DGCMG gimbal rates. As can be seen in Figure 10, the safe use of DGCMGs can be guaranteed by both the feedforward and feedback attitude tracking controllers. Because the feedforward controller makes use of the planning results directly, the gimbal rate trajectory in 


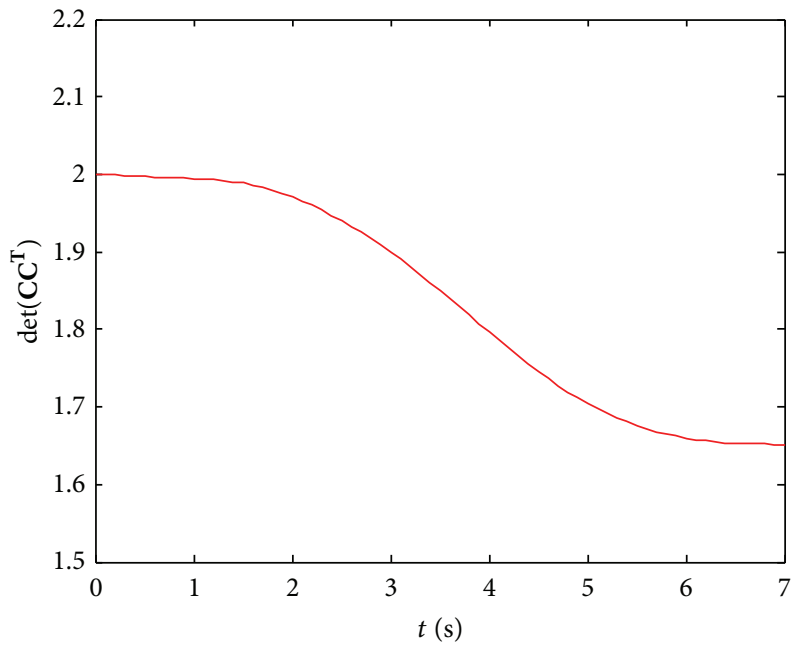

(a)

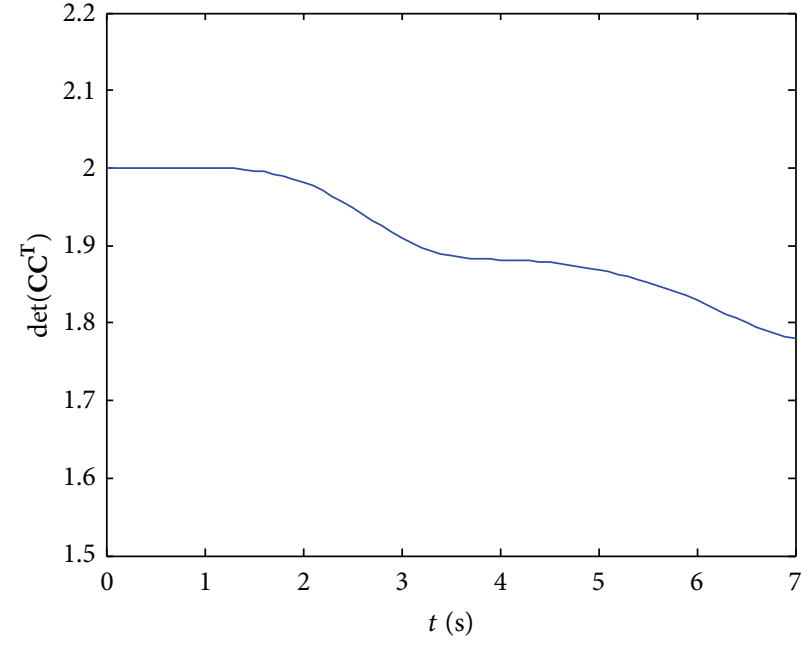

(b)

FIgURE 7: Time histories of singularity index. (a) Based on the path planning method in [7]. (b) Based on the path planning method in this paper.

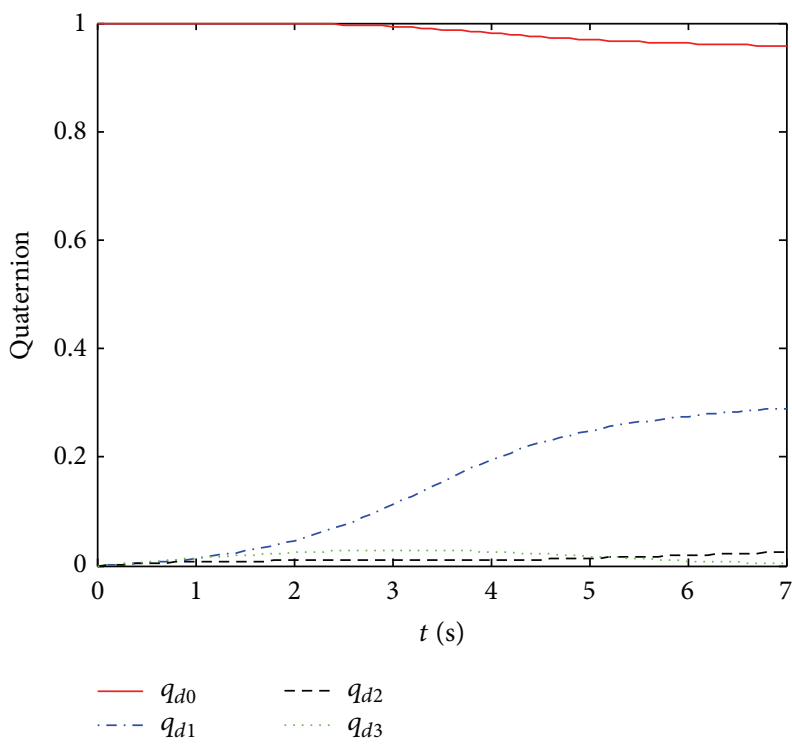

(a)

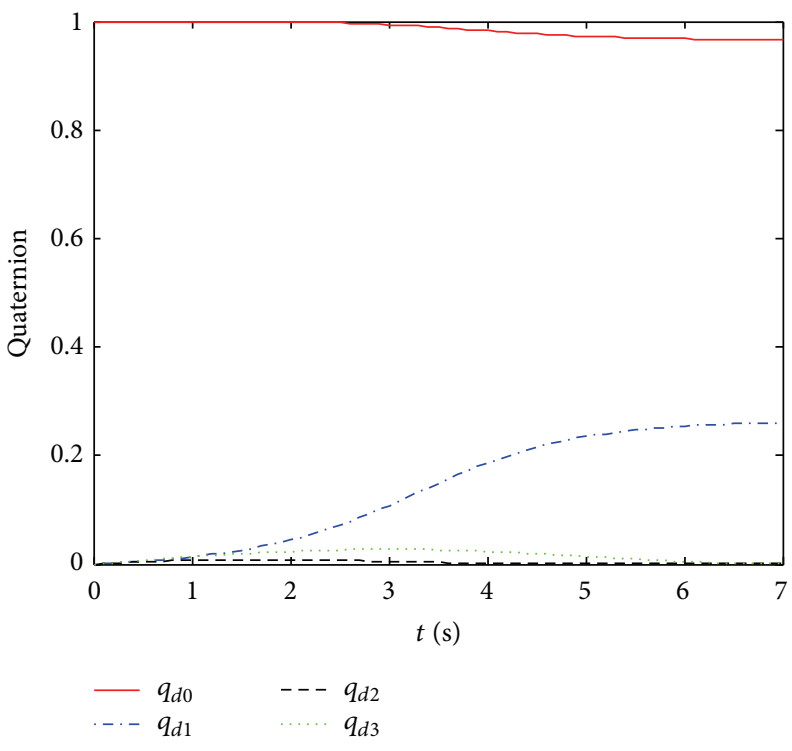

(b)

Figure 8: Time histories of quaternion. (a) Angular velocity with feedforward control. (b) Angular velocity with feedback control.

Figure 10(a) is the same as the planning path of gimbal rates. As shown in Figure 10(b), the DGCMG energy consumption increases owing to the feedback compensation. It is suggested that the robustness of the control system is obtained at the cost of increasing DGCMG energy consumption. Figure 11 illustrates gimbal angles of DGCMGs. The trajectories of gimbal angles vary smoothly during the whole maneuver time.

\section{Conclusions}

Considering the energy consumption and terminal state errors of double-gimbal control moment gyroscopes at the same time, the path planning method based on an improved FBA is proposed for planning a near optimal path with low energy consumption and smoothly varying DGCMG gimbal rates. The feedforward control using the above path can prevent the DGCMGs from encountering the singularity, and the rapid actuation of DGCMGs is guaranteed. In order to improve the search efficiency of the Fourier coefficients and reduce the computational time of the path planning, a choosing method of the Fourier coefficients is put forward according to the satellite maneuver characteristic. Owing to the fact that the feedforward control cannot acquire the robustness, a feedback controller based on the path planning is introduced. The forward control inputs 


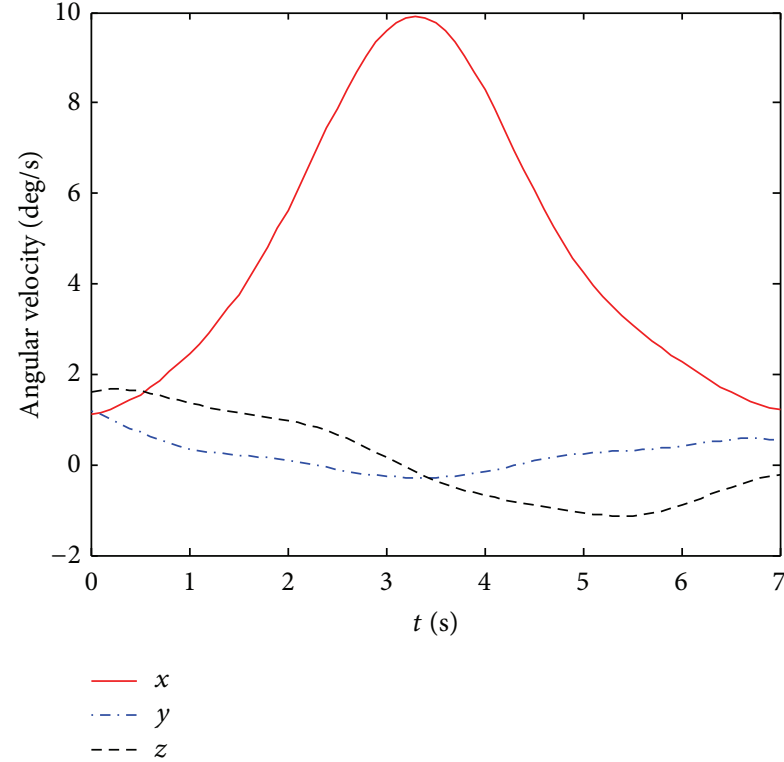

(a)

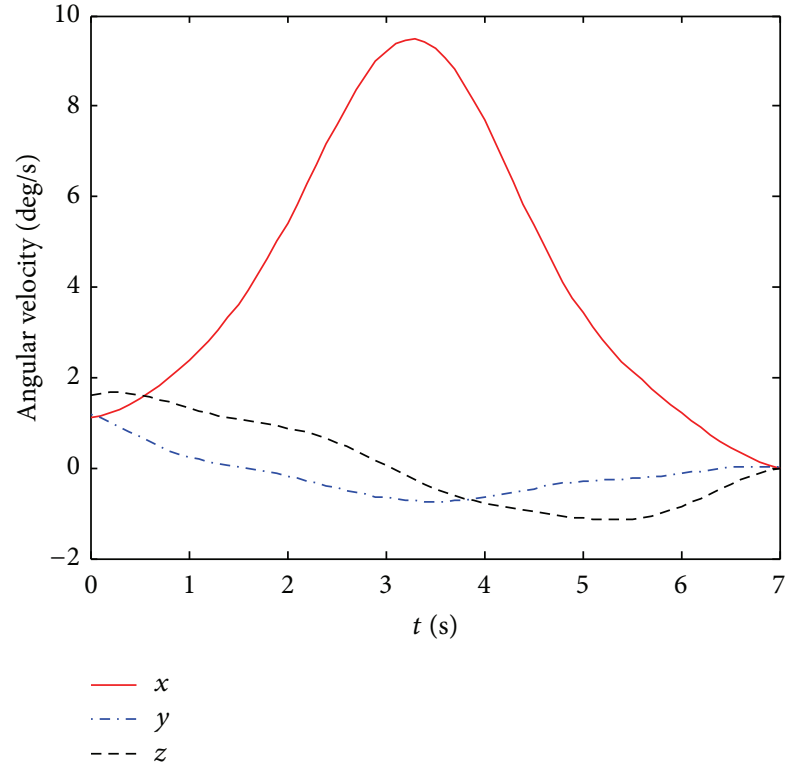

(b)

FIGURE 9: Time histories of attitude angular velocity. (a) Angular velocity with feedforward control. (b) Angular velocity with feedback control.

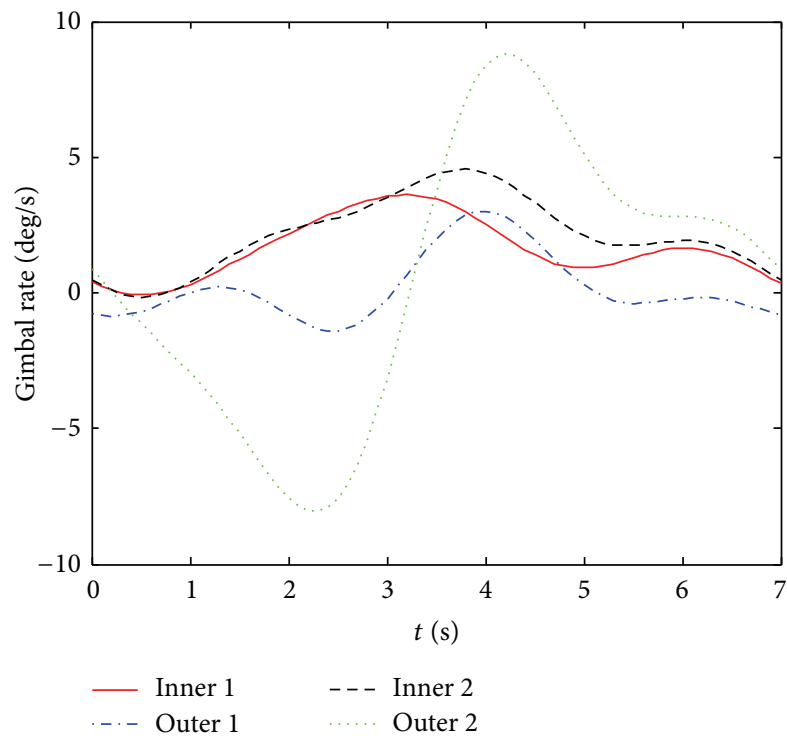

(a)

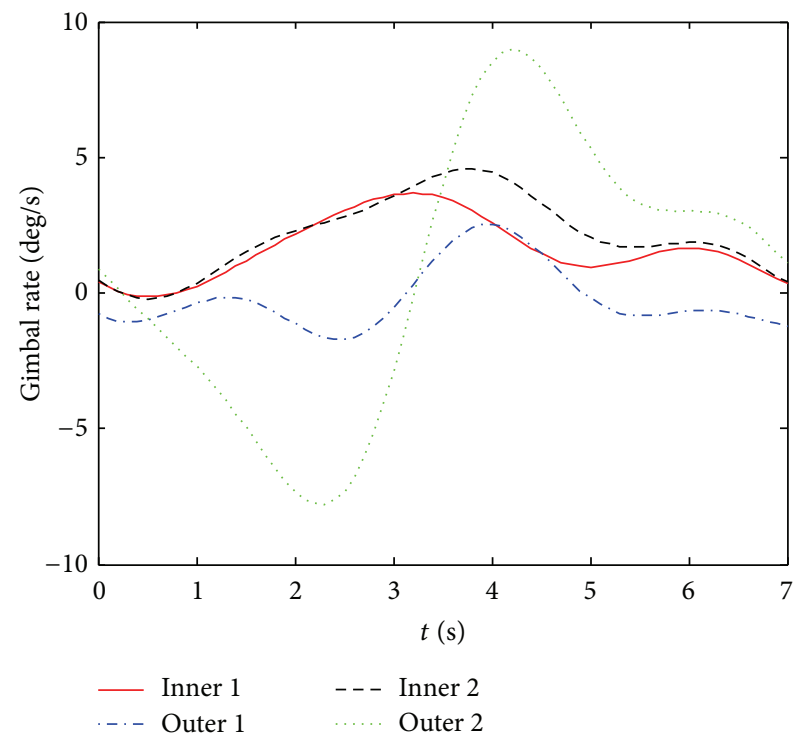

(b)

Figure 10: Time histories of gimbal rate. (a) Gimbal rates with feedforward control. (b) Gimbal rates with feedback control.

of the feedback controller are the planning gimbal rates, and the slide mode control method is used to compute the feedback control inputs. Thus, the accuracy of attitude control system is improved notably. Finally, the effectiveness of the proposed path planning method and attitude tracking feedback controller is validated by simulations. Comparing with the path planning method in [7], the proposed path planning method is advantageous in terms of the initial gimbal rates, maximal gimbal rates, and energy consumption of DGCMGs.
Moreover, the computational time of path planning is much shorter than that of [7] and thus is benefiting to the satellite attitude agile maneuver.

\section{Conflict of Interests}

The authors declare that there is no conflict of interests regarding the publication of this paper. 


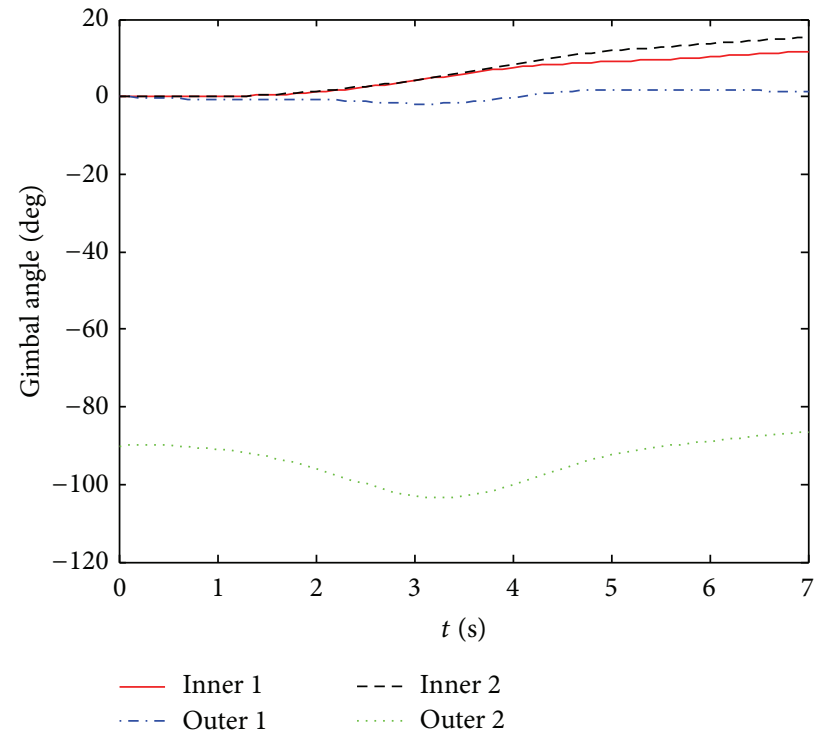

(a)

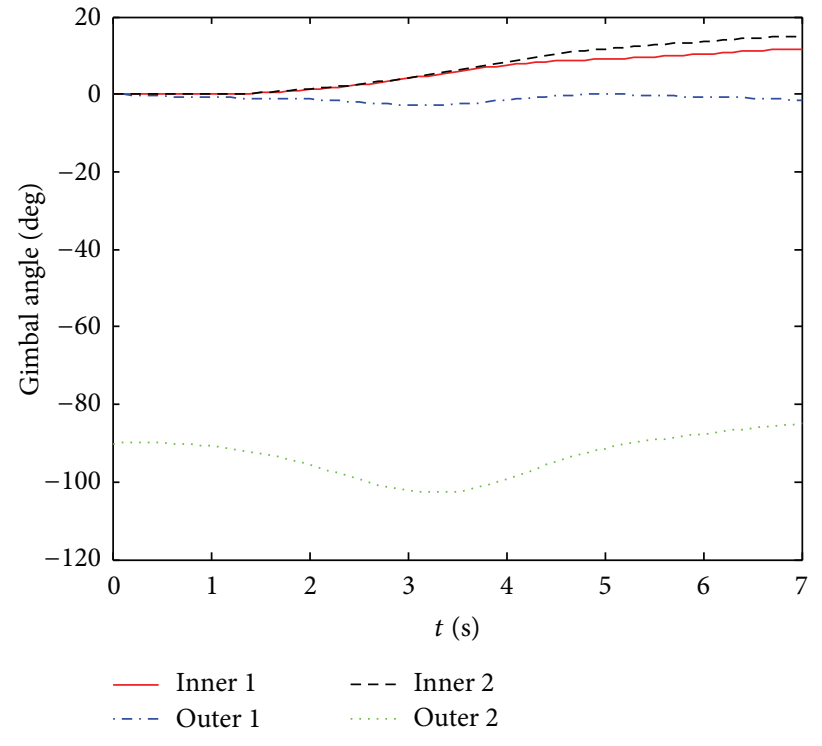

(b)

FIgURE 11: Time histories of gimbal angle. (a) Gimbal angles with feedforward control. (b) Gimbal angles with feedback control.

\section{Acknowledgments}

This research has been supported by National Natural Science Foundation of China under Grant 61121003, the National Basic Research Program (973 Program) of China under Grant 2009CB2400101C, and National Civil Aerospace Preresearch Project.

\section{References}

[1] J. Y. Zhou and D. Zhou, "Spacecraft attitude control with double-gimbaled control moment gyroscopes," in Proceedings of the IEEE International Conference on Robotics and Biomimetics (ROBIO '07), pp. 1557-1562, December 2007.

[2] S. Kasai, H. Kojima, and M. Satoh, "Spacecraft attitude maneuver using two single-gimbal control moment gyros," Acta Astronautica, vol. 84, pp. 88-98, 2013.

[3] T. Meng and S. Matunaga, "Modified singular-direction avoidance steering for control moment gyros," Journal of Guidance, Control, and Dynamics, vol. 34, no. 6, pp. 1915-1919, 2011.

[4] H. F. Kennel, "Steering law for parallel mounted doublegimbaled control moment gyros," Tech. Rep. NASA-TM-82390, NASA Marshall Space Flight Center, Huntsville, Ala, USA, 1981.

[5] G. Liu, C. J. Li, G. F. Ma, and J. Huang, "Time efficient controller design for satellite attitude maneuvers using SGCMG," Acta Aeronautica et Astronautica Sinica, vol. 32, no. 10, pp. 1905-1913, 2011 (Chinese).

[6] I. M. Ross and M. Karpenko, "A review of pseudospectral optimal control: from theory to flight," Annual Reviews in Control, vol. 36, no. 2, pp. 182-197, 2012.

[7] P. L. Cui and F. Y. Liu, "Attitude path planning for agile satellite based on improved genetic algorithm," Electronics Optics \& Control, vol. 19, no. 12, pp. 23-28, 2012 (Chinese).

[8] R. Kala, "Multi-robot path planning using co-evolutionary genetic programming," Expert Systems with Applications, vol. 39, no. 3, pp. 3817-3831, 2012.
[9] Y. Kusuda and M. Takahashi, "Feedback control with nominal inputs for agile satellites using control moment gyros," Journal of Guidance, Control, and Dynamics, vol. 34, no. 4, pp. 1209-1218, 2011.

[10] Y. Kusuda, M. Takahashi, and K. Yoshida, "Energy optimal path planning of satellite attitude maneuver using control moment gyros," in Proceedings of the 59th International Astronautical Congress, IAC Paper C1, Glasgow, UK, 2008. 


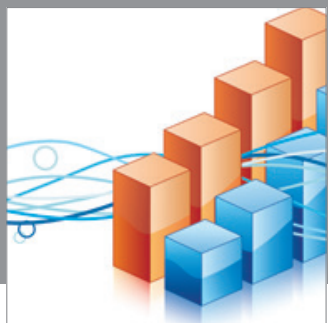

Advances in

Operations Research

mansans

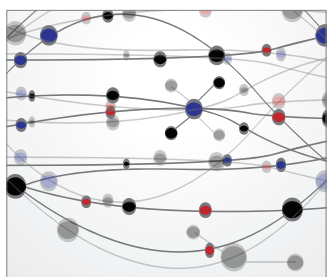

The Scientific World Journal
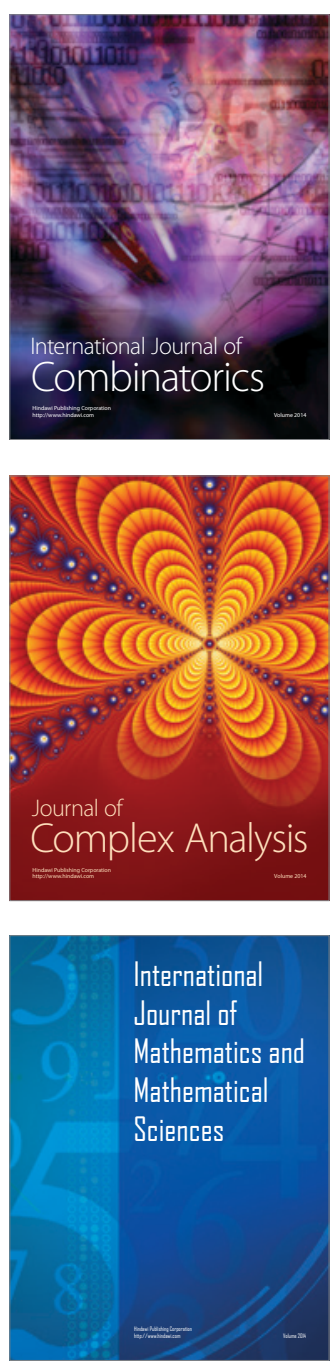
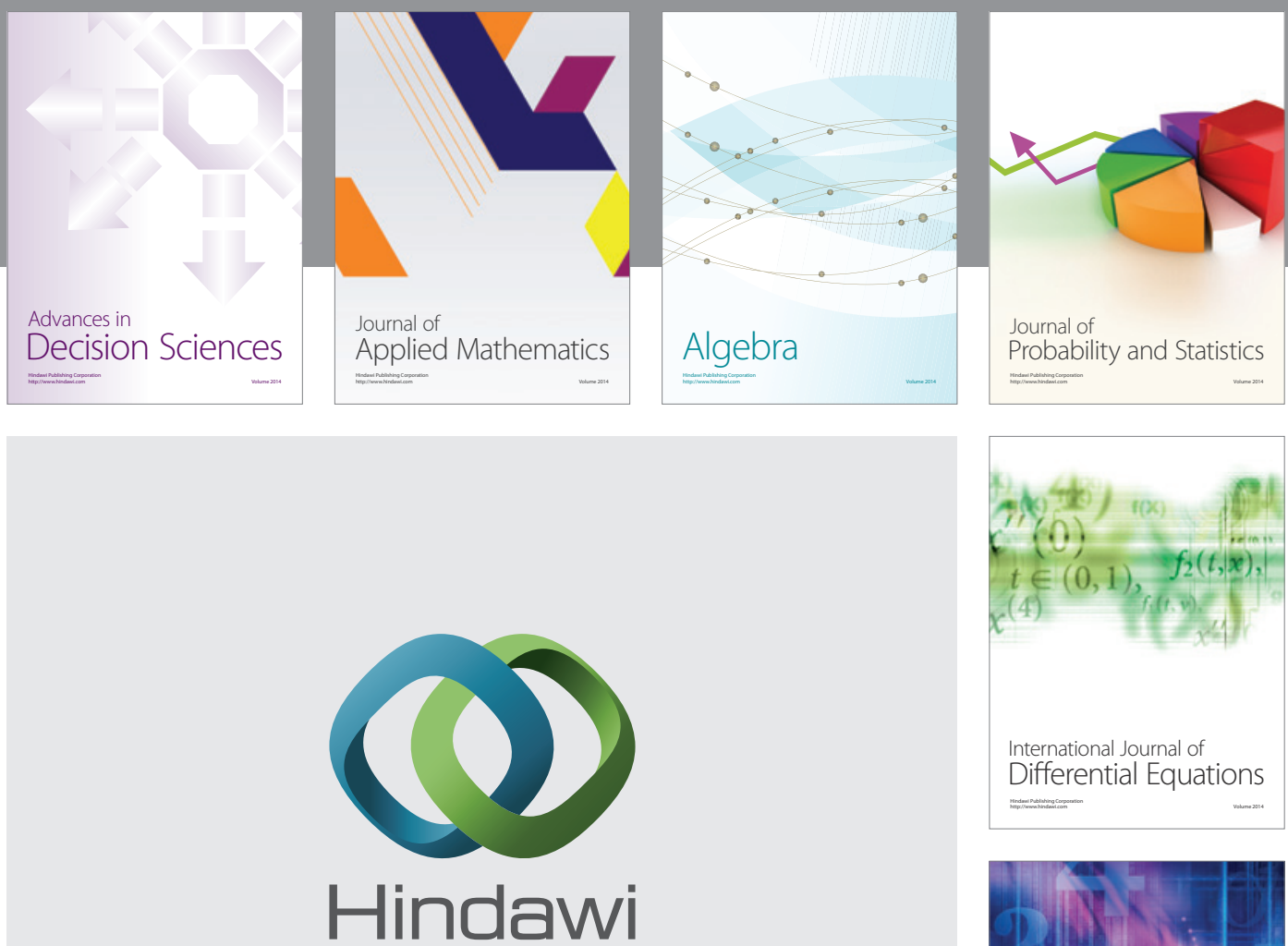

Submit your manuscripts at http://www.hindawi.com
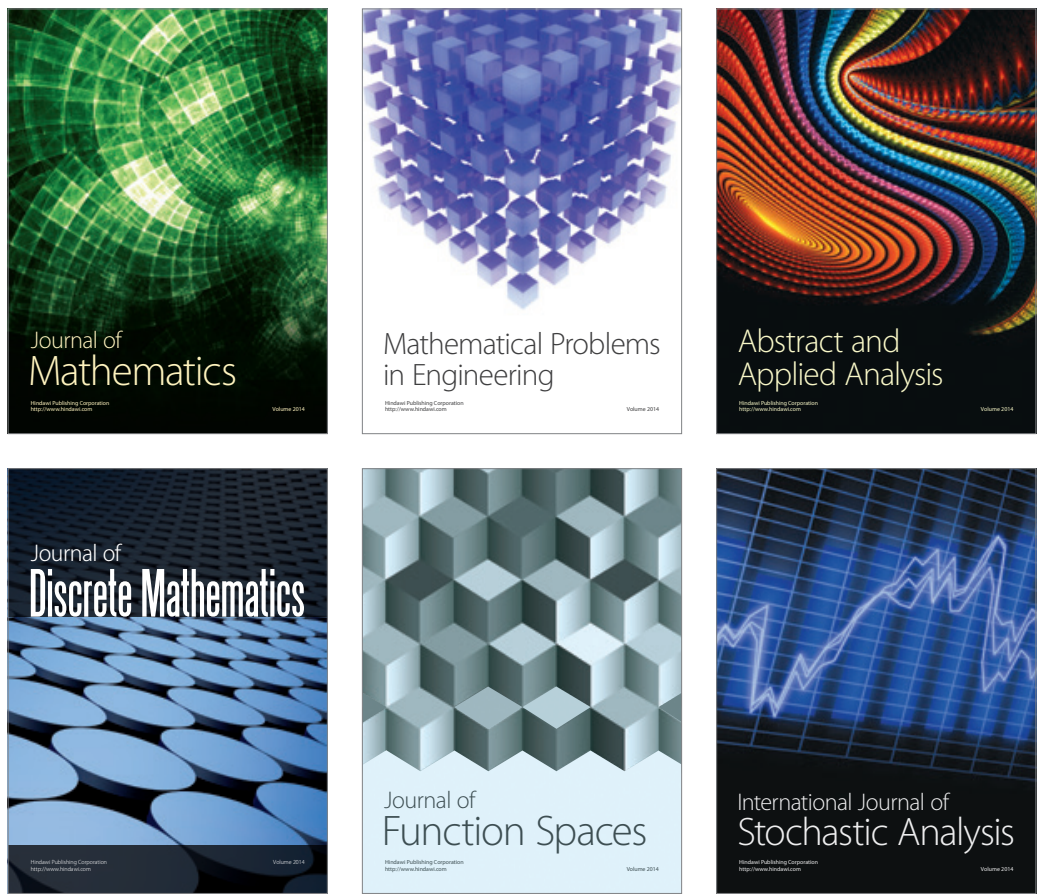

Journal of

Function Spaces

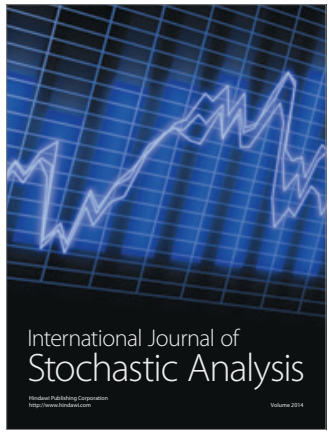

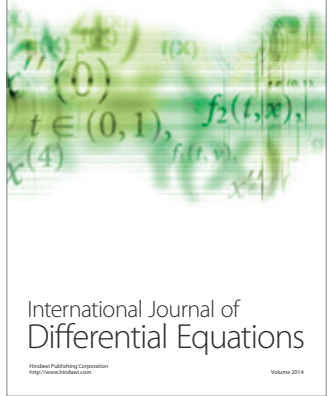
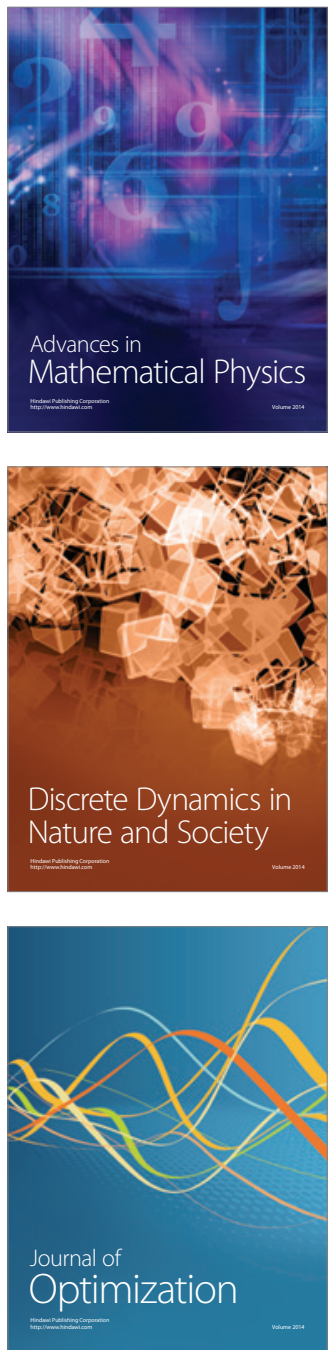